\title{
A Class of Generalized Evolutionary Problems Driven by Variational Inequalities and Fractional Operators
}

\author{
Stanisław Migórski ${ }^{1,2} \cdot$ Shengda Zeng ${ }^{3}$ \\ Received: 20 February 2018 / Accepted: 18 November 2018 /Published online: 29 November 2018 \\ (C) The Author(s) 2018
}

\begin{abstract}
This paper is devoted to a generalized evolution system called fractional partial differential variational inequality which consists of a mixed quasi-variational inequality combined with a fractional partial differential equation in a Banach space. Invoking the pseudomonotonicity of multivalued operators and a generalization of the Knaster-Kuratowski-Mazurkiewicz theorem, first, we prove that the solution set of the mixed quasi-variational inequality involved in system is nonempty, closed and convex. Next, the measurability and upper semicontinuity for the mixed quasi-variational inequality with respect to the time variable and state variable are established. Finally, the existence of mild solutions for the system is delivered. The approach is based on the theory of operator semigroups, the Bohnenblust-Karlin fixed point principle for multivalued mappings, and theory of fractional operators.
\end{abstract}

Keywords Fractional partial differential variational inequalities - Caputo derivative . Knaster-Kuratowski-Mazurkiewicz theorem · Bohnenblust-Karlin fixed point principle . $\phi$-pseudomonotonicity $\cdot$ Mixed quasi-variational inequalities

Mathematics Subject Classification (2010) 47J20 - 49J40 · 35J88 · 26A33 · 34A08

This project has received funding from the European Union's Horizon 2020 Research and Innovation Programme under the Marie Skł odowska-Curie grant agreement No. 823731 - CONMECH. It is supported by the National Science Center of Poland under Maestro Project No.

UMO-2012/06/A/ST1/00262, and National Science Center of Poland under Preludium Project No. 2017/25/N/ST1/00611. The first author is also supported by Qinzhou University Project No. 2018KYQD06, and the International Project co-financed by the Ministry of Science and Higher Education of Republic of Poland under Grant No. 3792/GGPJ/H2020/2017/0.

Shengda Zeng

zengshengda@163.com; shengdazeng@gmail.com; shdzeng@hotmail.com

Stanisław Migórski

stanislaw.migorski@uj.edu.pl

1 College of Sciences, Qinzhou University, Qinzhou, Guangxi 535000, People's Republic of China

2 Chair of Optimization and Control, Jagiellonian University in Krakow, ul. Lojasiewicza 6, 30348, Krakow, Poland

3 Faculty of Mathematics and Computer Science, Jagiellonian University in Krakow, ul. Lojasiewicza 6, 30348, Krakow, Poland 


\section{Introduction}

The fractional calculus, as a natural generalization of the classical integer order calculus, has been of great interest recently. Since fractional order derivatives hold local properties and memory effects, they can accurately describe many phenomena, for example, in electrodynamics, biotechnology, aerodynamics, distributed propeller design and control of dynamical systems, see [13, 16, 26, 28, 32, 39, 40, 42].

The notion of differential variational inequalities ((DVIs), for short) was introduced and systematically studied by Pang-Stewart [25] in Euclidean spaces. Since then, many researchers are attracted to boost the development of theory and applications of DVIs. The DVIs can be seen a useful tool to represent models involving both dynamics and constraints in the form of inequalities, and arise in many applied problems, for instance, electrical circuits with ideal diodes, Coulomb friction problems for contacting bodies, economical dynamics and related problems such as dynamic traffic networks, see cf. $[4,10,35]$. The most representative results are: Liu-Loi-Obukhovskii [19] who studied the existence and global bifurcation for periodic solutions of a class of differential variational inequalities in a finite dimensional space by applying the topological degree theory for multivalued maps and the method of guiding functions, Gwinner [8] who explored a stability result of a new class of differential variational inequalities by using the monotonicity method and the technique of the Mosco convergence, and Chen-Wang [5] who investigated the dynamic Nash equilibrium problems involved in a decision process with multiple players which had the formulation of differential mixed quasi-variational inequalities under the suitable conditions. For more details on this topics in finite dimensional spaces the reader may consult $[9,21,25,27,29]$ and the references therein.

It is noteworthy that all aforementioned works were considered only in finite dimensional spaces. This seriously limits their scope of applications since in many realistic problems in engineering, operations research, economical dynamics, physical sciences, etc. various phenomena are more accurately described by partial differential equations. Based on this motivation, recently, Liu-Zeng-Motreanu [18] and Liu-Migórski-Zeng [15] proved the existence of solutions for a class of differential mixed variational inequalities in Banach spaces by applying the theory of semigroups, the Filippov implicit function lemma, fixed point theorems for condensing multivalued operators, etc. After that, Migórski-Zeng [22] applied a temporally semi-discrete method based on the backward Euler difference scheme and a feedback iterative technique to address a new kind of problems, which consist of a hemivariational inequality of parabolic type combined with a nonlinear evolution equation in the framework of an evolution triple of spaces. In the meantime, Ke-Loi-Obukhovskii [12] firstly considered a differential variational inequality in finite dimensional spaces driven by fractional derivative operator. However, a natural question has been raised why there is a need to study the fractional differential variational inequalities. More recently, Zeng-LiuMigórski [41] has delivered a positive answer to this question. Actually, the authors in [41] applied the Rothe method combined with surjectivity of multivalued pseudomonotone operators and properties of the Clarke generalized gradient to establish existence of solutions to the following fractional differential hemivariational inequality in a Banach space

$$
\left\{\begin{array}{l}
\left.\left\langle A{ }_{0}^{C} D_{t}^{\alpha} u(t)\right)+B(u(t)), v\right\rangle+J^{0}(\beta(t), M u(t) ; M v) \geq\langle f(t), v\rangle \\
\quad \quad \text { for all } v \in V, \text { a.e. } t \in(0, T), \\
u(0)=u_{0}, \\
\beta^{\prime}(t)=g(t, M u(t), \beta) \text { for a.e. } t \in(0, T), \\
\beta(0)=\beta_{0} .
\end{array}\right.
$$


Then, they have used the abstract results to study a quasistatic frictional contact problem for viscoelastic materials with adhesion effect in which the friction and contact conditions are described by the Clarke generalized gradient of nonconvex and nonsmooth functionals, and the constitutive relation is modeled by the fractional Kelvin-Voigt law.

Let $E$ and $E_{1}$ be two real reflexive, separable Banach spaces, $K$ be a nonempty, closed and convex subset of $E, 0<T<\infty$, and $A: D(A) \subset E_{1} \rightarrow E_{1}$ be the infinitesimal generator of a $C_{0}$-semigroup $e^{A t}$ in $E_{1}$ such that

$$
\sup _{t \in[0, T]}\left\|e^{A t}\right\| \leq M_{A} \text { for some } M_{A}>0 .
$$

In this paper, given functions $\phi: K \rightarrow \overline{\mathbb{R}}:=(-\infty,+\infty], G: K \rightarrow P\left(E^{*}\right), B:[0, T] \times$ $E_{1} \rightarrow \mathcal{L}\left(E, E_{1}\right), f:[0, T] \times E_{1} \rightarrow E_{1}$, and $g:[0, T] \times E_{1} \rightarrow E^{*}$, we consider the following generalized evolution system

$$
(\text { FPDVI }) \begin{cases}{ }^{C} D_{t}^{\alpha} x(t)=A x(t)+f(t, x(t))+B(t, x(t)) u(t) & \text { a.e. } t \in(0, T) \\ u(t) \in S O L(K, g(t, x(t))+G(\cdot), \phi) & \text { a.e. } t \in(0, T) \\ x(0)=x_{0} . & \end{cases}
$$

Here, the operator ${ }^{C} D_{t}^{\alpha}$ stands for the classical fractional derivative operator in the sense of Caputo (see Definition 2.3 below) and the set $S O L(K, g(t, x(t))+G(\cdot), \phi) \subset E$ represents the solution set of the following mixed quasi-variational inequality ((MQVI), for short): given $t \in[0, T]$ and $x(t) \in E_{1}$, find $u(t) \in K$ and $u^{*} \in G(u(t))$ such that

$$
\left\langle u^{*}+g(t, x(t)), v-u(t)\right\rangle+\phi(v)-\phi(u(t)) \geq 0 \text { for all } v \in K .
$$

Moreover, to highlight the level of generalization of our problem (1.1), we present below several its particular cases.

(1) If $E=\mathbb{R}^{m}, E_{1}=\mathbb{R}^{n}, f=0, \phi=0$ and $G: K \rightarrow \mathbb{R}^{m}$ is a single valued mapping, then (FPDVI) (1.1) reduces to the following linear fractional differential variational inequality

$$
\left\{\begin{array}{l}
{ }^{C} D_{t}^{\alpha} x(t)=A x(t)+B(t, x(t)) u(t) \text { a.e. } t \in(0, T) \\
u(t) \in S O L(K, g(t, x(t))+G(\cdot)) \text { a.e. } t \in(0, T) \\
x(0)=x_{0},
\end{array}\right.
$$

which has been explored by Ke-Loi-Obukhovskii in [12].

(2) If $\alpha=1, A=0, E=\mathbb{R}^{m}, E_{1}=\mathbb{R}^{n}$, and $\phi=0$, then (FPDVI) (1.1) becomes the following differential variational inequality

$$
\left\{\begin{array}{l}
x^{\prime}(t)=f(t, x(t))+B(t, x(t)) u(t) \text { a.e. } t \in(0, T) \\
u(t) \in S O L(K, g(t, x(t))+G(\cdot)) \text { a.e. } t \in(0, T) \\
x(0)=x_{0}
\end{array}\right.
$$

which has been sstudied by Wang-Li-Li-Huang in [36].

(3) If $\alpha=1, A=0, E=\mathbb{R}^{m}, E_{1}=\mathbb{R}^{n}$, and $G: K \rightarrow \mathbb{R}^{m}$ is a single valued mapping, then (FPDVI) (1.1) turns into the following differential mixed variational inequality

$$
\begin{cases}x^{\prime}(t)=f(t, x(t))+B(t, x(t)) u(t) & \text { a.e. } t \in(0, T) \\ u(t) \in S O L(K, g(t, x(t))+G(\cdot), \phi) & \text { a.e. } t \in(0, T) \\ x(0)=x_{0}, & \end{cases}
$$


which has been investigated by Li-Huang-O'Regan in [14]. For other special cases, the reader can consult $[17,23,31,33,34,36,37]$.

The paper is structured as follows. Section 2 recalls some basic definitions and preliminary facts needed in the sequel. In Section 3, we establish the upper semicontinuity and measurability of the solution set of (MQVI) with respect to the time variable $t$ and state variable $x$. Based on results of Section 3, in Section 4, we explore the existence of mild solutions for (FPDVI) via applying Bohnenblust-Karlin fixed point principle, theory of operator semigroups, and theory of fractional operators. Finally, we make a conclusion.

\section{Notation and preliminary results}

In this section, we recall briefly the necessary background material for self-contained presentation of our study. Throughout the rest of the paper, we denote by $P(Y)$ all nonempty subsets of a topological space $Y$, and use the notations

$$
\begin{aligned}
& C(Y):=\{D \in P(Y) \mid D \text { is closed }\} \\
& K(Y):=\{D \in P(Y) \mid D \text { is compact }\} \\
& C(b) v(Y):=\{D \in P(Y) \mid D \text { is closed (bounded) and convex }\} .
\end{aligned}
$$

Let $\left(E_{1},\|\cdot\|_{E_{1}}\right)$ be a Banach space with its dual $E_{1}^{*}$ and $A: D(A) \subset E_{1} \rightarrow E_{1}$ be the infinitesimal generator of $C_{0}$-semigroup $\left\{e^{A t}, t \geq 0\right\}$. We denote by $C\left(J ; E_{1}\right)$ the space of continuous functions from $J:=[0, T]$ to $E_{1}$ with norm $\|x\|_{C}:=\sup _{t \in J}\|x(t)\|_{E_{1}}$ and by $A C\left(J ; E_{1}\right)$ the space of all absolutely continuous functions on $J$. We start with the following definitions about fractional operators, for more details, we refer to the references [13, $26,28]$.

Definition 2.1 Let $f \in L^{1}\left(0, T ; E_{1}\right)$ and $\alpha>0$. The Riemann-Liouville fractional integral of order $\alpha>0$ of $f$ is given by

$$
{ }_{0} I_{t}^{\alpha} f(t)=\frac{1}{\Gamma(\alpha)} \int_{0}^{t}(t-s)^{\alpha-1} f(s) d s \text { for a.e. } t \in(0, T),
$$

where $\Gamma(\cdot)$ is the well-known Gamma function defined by

$$
\Gamma(\alpha)=\int_{0}^{\infty} t^{\alpha-1} e^{-t} d t .
$$

Definition 2.2 The Riemann-Liouville fractional derivative of order $n-1<\alpha \leq n$ of $f:[0, T] \rightarrow E_{1}$ is defined by

$$
{ }^{R} D_{t}^{\alpha} f(t)=\frac{1}{\Gamma(n-\alpha)}\left(\frac{d}{d t}\right)^{n} \int_{0}^{t}(t-s)^{n-\alpha-1} f(s) d s \text { for a.e. } t \in(0, T) .
$$

Definition 2.3 The Caputo fractional derivative of order $n-1<\alpha \leq n$ of $f:[0, T] \rightarrow E_{1}$ is defined as

$$
{ }^{C} D_{t}^{\alpha} f(t)={ }^{R} D_{t}^{\alpha}\left(f(t)-\sum_{k=0}^{n-1} \frac{t^{k}}{k !} f^{(k)}(0)\right) \text { for a.e. } t \in(0, T) .
$$

According to our previous work $[15,17,18,21]$, we give the definition of a mild solution. 
Definition 2.4 A pair of functions $(x, u)$, with $x \in C\left(0, T ; E_{1}\right)$ and $u:[0, T] \rightarrow K$ measurable, is said to be a mild solution of the problem (FPDVI) in (1.1), if

$$
\left\{\begin{array}{l}
x(t)=P_{\alpha}(t) x_{0}+\int_{0}^{t}(t-s)^{\alpha-1} Q_{\alpha}(t-s)[B(s, x(s)) u(s)+f(s, x(s))] d s \\
u(t) \in S O L(K, g(t, x(t))+G(\cdot), \phi)
\end{array}\right.
$$

for a.e. $t \in(0, T)$, where

$$
\begin{aligned}
& P_{\alpha}(t)=\int_{0}^{\infty} \xi_{\alpha}(\theta) e^{A\left(t^{\alpha} \theta\right)} d \theta, \\
& Q_{\alpha}(t)=\alpha \int_{0}^{\infty} \theta \xi_{\alpha}(\theta) e^{A\left(t^{\alpha} \theta\right)} d \theta, \\
& \xi_{\alpha}(\theta)=\frac{1}{\alpha} \theta^{-1-\frac{1}{\alpha}} \omega_{\alpha}\left(\theta^{-\frac{1}{\alpha}}\right) \geq 0, \\
& \omega_{\alpha}=\frac{1}{\pi} \sum_{n=1}^{\infty}(-1)^{n-1} \theta^{-\alpha n-1} \frac{\Gamma(\alpha n+1)}{n !} \sin (n \pi \alpha), \quad \theta \in(0, \infty) .
\end{aligned}
$$

Remark 2.5 The function $\xi_{\alpha}$ is usually called a probability density function on $(0, \infty)$, since it has the following properties

$$
\xi_{\alpha}(\theta) \geq 0 \text { for } \theta \in(0,+\infty) \text { with } \int_{0}^{\infty} \xi_{\alpha}(\theta) d \theta=1
$$

Moreover, by virtue of definition of $\xi_{\alpha}$, one has

$$
\int_{0}^{\infty} \theta^{\beta} \xi_{\alpha}(\theta) d \theta=\int_{0}^{\infty} \theta^{-\alpha \beta} \omega_{\alpha}(\theta) d \theta=\frac{\Gamma(1+\beta)}{\Gamma(1+\alpha \beta)} \text { for } \beta \in[0,1] .
$$

Next, we shall recall the following lemma, which reveals several important properties of $P_{\alpha}$ and $Q_{\alpha}$.

Lemma 2.6 [32, 42] Let $A: D(A) \rightarrow E_{1}$ be the infinitesimal generator of a strongly continuous semigroup $\left\{e^{A t}, t \geq 0\right\}$. If there is a constant $M_{A}>0$ such that $\sup _{t \geq 0}\left\|e^{A t}\right\| \leq$ $M_{A}$, then operators $P_{\alpha}$ and $Q_{\alpha}$ fulfill the following properties:

(i) for $t \geq 0$ given, $P_{\alpha}(t)$ and $Q_{\alpha}(t)$ are linear and bounded, more precisely, for any $x \in E_{1}$, we have

$$
\left\|P_{\alpha}(t) x\right\|_{E_{1}} \leq M_{A}\|x\|_{E_{1}} \text { and }\left\|Q_{\alpha}(t) x\right\|_{E_{1}} \leq \frac{M_{A}}{\Gamma(\alpha)}\|x\|_{E_{1}},
$$

(ii) both $\left\{P_{\alpha}(t), t \geq 0\right\}$ and $\left\{Q_{\alpha}(t), t \geq 0\right\}$ are strongly continuous;

(iii) if the semigroup $\left\{e^{A t}, t>0\right\}$ is compact, then for every $t>0$, the operators $P_{\alpha}(t)$ and $Q_{\alpha}(t)$ are also compact.

Let $X$ and $Y$ be two topological spaces. Recall that a multivalued mapping $F: X \rightarrow$ $P(Y)$ is called to be upper semicontinuous (u.s.c., for short) at point $x_{0} \in X$, if and only if for any open set $V \subset Y$ with $F\left(x_{0}\right) \subset V$, there exists a neighborhood $O\left(x_{0}\right)$ of $x_{0}$ such that $F(x) \subset V$ for all $x \in O\left(x_{0}\right)$. Moreover, the following theorem provides criteria to verify the upper semicontinuity of a multivalued mapping. 
Proposition 2.7 [24] Let $F: X \rightarrow P(Y)$, with $X$ and $Y$ topological spaces. The following statements are equivalent:

(i) $F$ is upper semicontinuous,

(ii) for each closed set $C \subset Y$, the set $F^{-}(C):=\{x \in X \mid F(x) \cap C \neq \emptyset\}$ is closed in $X$,

(iii) for each open set $O \subset Y$, the set $F^{+}(O):=\{x \in X \mid F(x) \subset O\}$ is open in $X$.

On the other hand, we also recall the concept of quasicompact operators.

Definition 2.8 [11, Definition 1.1.5] A multivalued mapping $F: X \rightarrow P(Y)$ is called to be

(i) compact, if its range $F(X)$ is a relatively compact set in $Y$,

(ii) quasicompact, if its restriction to any compact subset $A \subset X$ is compact.

Theorem 2.9 [11, Theorem 1.1.12] Let $X$ and $Y$ be metric spaces. If $F: X \rightarrow P(Y)$ be a closed and quasicompact multivalued mapping, then $F$ is upper semicontinuous.

Proposition 2.10 [3, Proposition 2] Let $E$ and $E_{1}$ be real Banach spaces and $D$ be a nonempty subset of $E_{1}$. Assume that $G: D \rightarrow P(E)$ is a multivalued mapping with weakly compact and convex values. Then $G$ is strongly-weakly u.s.c. if and only if $\left\{x_{n}\right\} \subset D$ with $x_{n} \rightarrow x_{0} \in D$ and $y_{n} \in G\left(x_{n}\right)$ implies that there exists a subsequence $\left\{y_{n_{k}}\right\}$ of $\left\{y_{n}\right\}$ such that $y_{n_{k}} \rightarrow y_{0} \in G\left(x_{0}\right)$.

Furthermore, we review the following Bohnenblust-Karlin fixed point theorem which is the key tool in one of our main.

Theorem 2.11 [2] Let $\mathbb{D}$ be a nonempty subset of a Banach space $X$, which is bounded, closed and convex. Suppose $G: \mathbb{D} \rightarrow P(X)$ is u.s.c. with closed, convex values, and such that $G(\mathbb{D}) \subseteq \mathbb{D}$ and $\overline{G(\mathbb{D})}$ is compact (i.e., $G(\mathbb{D})$ is a relatively compact set). Then $G$ has a fixed point.

We conclude this section with the well-known F-KKM theorem by Ky Fan [7].

Lemma 2.12 [7] Let $K$ be a nonempty subset of a Hausdorff topological vector space $E$ and let $G: K \rightarrow P(E)$ be a multivalued mapping with the following properties:

(a) $G$ is a KKM mapping, that is, for any $\left\{v_{1}, v_{2}, \ldots, v_{n}\right\} \subset K$, one has that its convex hull co $\left\{v_{1}, v_{2}, \ldots, v_{n}\right\}$ is contained in $\bigcup_{i=1}^{n} G\left(v_{i}\right)$,

(b) for every $v \in K, G(v)$ is closed in $E$,

(c) for some $v_{0} \in K, G\left(v_{0}\right)$ is compact in $E$.

Then, we have $\bigcap_{v \in K} G(v) \neq \emptyset$.

\section{Mixed quasi-variational inequalities for (FPDVI)}

Let $\left(E,\|\cdot\|_{E}\right)$ be a real reflexive Banach space with its dual $E^{*}$, and $K$ be a nonempty, closed and convex subset of $E$. At the beginning of this section, we introduce the generalized $\phi$-pseudomonotonicity for multivalued mappings. 
Definition 3.1 Let $G: K \rightarrow P\left(E^{*}\right)$ be a multivalued mapping and $\phi: K \rightarrow \overline{\mathbb{R}}$. The map $G$ is said to be $\phi$-pseudomonotone on $K$, if for each $u, v \in K$ and $u^{*} \in G(u)$, it holds

$\left\langle u^{*}, v-u\right\rangle+\phi(v)-\phi(u) \geq 0 \quad \Longrightarrow \quad\left\langle v^{*}, v-u\right\rangle+\phi(v)-\phi(u) \geq 0$ for all $v^{*} \in G(v)$.

Remark 3.2 It is obvious that if $G$ is a pseudomonotone monotone operator, i.e., $\phi$ pseudomonotone with $\phi=0$ (in particular, a monotone operator), then it is also $\phi$-pseudomonotone for any function $\phi$. The converse is not true, in general. In fact, there have many counterexamples that can indicate this fact. For the case, if $G$ is a single-valued operator, we can refer Example 1 and 2 in [20]. For the case, when $G$ is a multivalued operator, here, we provide the following example to show this assertion.

Example 1 ( $G$ is a multivalued operator) Let $E=\mathbb{R}, K=[2,5], \phi(x)=x^{2}$ and

$$
G(x)= \begin{cases}{[1,2],} & \text { if } x \in[2,3] \\ \{-2 x+7\}, & \text { if } x \in[3,4] . \\ {[-3,-1],} & \text { if } x \in[4,5]\end{cases}
$$

Chosing $x=\frac{5}{2}$ and $y=\frac{9}{2}$, we have $\left\langle x^{*}, y-x\right\rangle \geq 2 \geq 0$ for all $x^{*} \in G\left(\frac{5}{2}\right)=[1,2]$ and

$$
\left\langle y^{*}, y-x\right\rangle \leq-2<0 \text { for all } y^{*} \in G\left(\frac{9}{2}\right)=[-3,-1] .
$$

So $G$ is not pseudomonotone in $K$. But, if $u, v \in[2,5]$ satisfy

$$
0 \leq\left\langle u^{*}, v-u\right\rangle+\phi(v)-\phi(u)=(v-u)\left(u+v+u^{*}\right) \text { for all } u^{*} \in G(u),
$$

then $v \geq u$, which leads to

$$
\left\langle v^{*}, v-u\right\rangle+\phi(v)-\phi(u)=(v-u)\left(u+v+v^{*}\right) \geq 0 \text { for all } v^{*} \in G(v) .
$$

Therefore, $G$ is $\phi$-pseudomonotone.

Now, we turn our attention to the following mixed quasi-variational inequality ((MQVI), for short): find $u \in K$ such that there exists $u^{*} \in G(u)$ and

$$
\left\langle u^{*}+H, v-u\right\rangle+\phi(v)-\phi(u) \geq 0 \text { for all } v \in K,
$$

where $H \in E^{*}, \phi: K \rightarrow \overline{\mathbb{R}}:=\mathbb{R} \cup\{+\infty\}$ is a proper, convex and lower semicontinuous function, and $G: K \rightarrow P\left(E^{*}\right)$ is a multivalued mapping. In what follows, we denote

$$
\operatorname{SOL}(K, G(\cdot)+H, \phi):=\{u \in K \mid u \text { is a solution to problem (3.1) }\} \subset E
$$

to be the solution set of (MQVI) in (3.1).

We start with the following theorem.

Theorem 3.3 Let $K$ be a nonempty, closed and convex subset of a real reflexive Banach space E. Assume that

$\left(A_{1}\right) \quad G: K \rightarrow P\left(E^{*}\right)$ is upper semicontinuous with compact values, and $G(\cdot)+H$ is $\phi$-pseudomonotone,

$\left(A_{2}\right) \quad$ if the set $K$ is unbounded in $E$, then there exists $\widetilde{v} \in K \cap D(\phi)$ such that

$$
\lim _{u \in K,\|u\|_{E} \rightarrow+\infty} \frac{\inf _{u^{*} \in G(u)}\left\langle u^{*}, u-\widetilde{v}\right\rangle+\phi(u)-\phi(\widetilde{v})}{\|u\|_{E}}=+\infty .
$$


Then, the solution set $\operatorname{SOL}(K, G(\cdot)+H, \phi)$ of $(M Q V I)$ in (3.1) is nonempty, closed and convex in $E$.

Proof First, we will show that the set $\operatorname{SOL}(K, G(\cdot)+H, \phi)$ is closed. Let $\left\{u_{n}\right\} \subset$ $\operatorname{SOL}(K, G(\cdot)+H, \phi)$ be such that $u_{n} \rightarrow u$ in $K$. Then, for each $n \in \mathbb{N}$, there exists $u_{n}^{*} \in G\left(u_{n}\right)$ such that

$$
\left\langle u_{n}^{*}+H, v-u_{n}\right\rangle+\phi(v)-\phi\left(u_{n}\right) \geq 0 \text { for all } v \in K .
$$

Since $G$ is upper semicontinuous with compact values and $u_{n} \rightarrow u$ in $K$, there is a subsequence $\left\{u_{n_{k}}^{*}\right\}$ of $\left\{u_{n}^{*}\right\}$ such that $u_{n_{k}}^{*} \rightarrow u^{*} \in G(u)$ (more details one can see [30]). In addition, the lower semicontinuity of $\phi$ guarantees that

$$
\left\langle u^{*}+H, v-u\right\rangle+\phi(v)-\phi(u) \geq 0 \text { for all } v \in K .
$$

This means that $u \in \operatorname{SOL}(K, G(\cdot)+H, \phi)$, thus $\operatorname{SOL}(K, G(\cdot)+H, \phi)$ is a closed subset of $K$.

Next, we shall verify the convexity of $\operatorname{SOL}(K, G(\cdot)+H, \phi)$. Let $u_{1}, u_{2} \in$ $\operatorname{SOL}(K, G(\cdot)+H, \phi)$. So, there exist $u_{1}^{*} \in G\left(u_{1}\right)$ and $u_{2}^{*} \in G\left(u_{2}\right)$ such that

$$
\left\langle u_{i}^{*}+H, v-u_{i}\right\rangle+\phi(v)-\phi\left(u_{i}\right) \geq 0 \text { for all } v \in K, i=1,2 .
$$

It follows from $\phi$-pseudomonotonicity of $G+H$ that

$$
\left\langle v^{*}+H, v-u_{i}\right\rangle+\phi(v)-\phi\left(u_{i}\right) \geq 0 \text { for all } v^{*} \in G(v) \text { and } v \in K, \quad i=1,2 .
$$

For any $\lambda \in(0,1)$, we denote $u_{\lambda}=\lambda u_{1}+(1-\lambda) u_{2}$. From the convexity of $\phi$, we have

$$
\begin{aligned}
& \left\langle v^{*}+H, v-u_{\lambda}\right\rangle+\phi(v)-\phi\left(u_{\lambda}\right) \geq \lambda\left[\left\langle v^{*}+H, v-u_{1}\right\rangle+\phi(v)-\phi\left(u_{1}\right)\right] \\
& \quad+(1-\lambda)\left[\left\langle v^{*}+H, v-u_{2}\right\rangle+\phi(v)-\phi\left(u_{2}\right)\right] \geq 0
\end{aligned}
$$

for all $v^{*} \in G(v)$ and all $v \in K$. Moreover, for any $v \in K$ and $t \in(0,1]$, inserting $v=v_{t}:=t v+(1-t) u_{\lambda} \in K$ into (3.3), we get

$$
\left\langle v_{t}^{*}+H, v_{t}-u_{\lambda}\right\rangle+\phi\left(v_{t}\right)-\phi\left(u_{\lambda}\right) \geq 0 \text { for all } v_{t}^{*} \in G\left(v_{t}\right)
$$

Hence, by the convexity of $\phi$, we find

$$
\left\langle v_{t}^{*}+H, v-u_{\lambda}\right\rangle+\phi(v)-\phi\left(u_{\lambda}\right) \geq 0 \text { for all } v_{t}^{*} \in G\left(v_{t}\right) .
$$

Having in mind that $G$ is u.s.c with compact values, and taking the limit as $t \rightarrow 0^{+}$, in the above inequality, without any loss of generality, we may assume that $v_{t}^{*} \rightarrow u_{\lambda}^{*} \in G\left(u_{\lambda}\right)$. Therefore, we easily get

$$
\left\langle u_{\lambda}^{*}+H, v-u_{\lambda}\right\rangle+\phi(v)-\phi\left(u_{\lambda}\right) \geq 0 \text { for all } v \in K .
$$

Hence, $u_{\lambda} \in \operatorname{SOL}(K, G(\cdot)+H, \phi)$, and thus $\operatorname{SOL}(K, G(\cdot)+H, \phi)$ is convex.

It remains to prove the nonemptiness of $\operatorname{SOL}(K, G(\cdot)+H, \phi)$. To do so, we first assume that $K$ is bounded. We consider the multivalued mapping $Q: K \rightarrow P(K)$ defined by

$$
Q(v):=\left\{u \in K \mid \inf _{v^{*} \in G(v)}\left\langle v^{*}+H, v-u\right\rangle+\phi(v)-\phi(u) \geq 0\right\} \text { for } v \in K .
$$

We readily get $v \in Q(v)$ for all $v \in K$, i.e., $Q$ is well-defined. Next, we can demonstrate that $Q(v)$ is weakly closed for each $v \in K$. Indeed, let $\left\{u_{n}\right\} \subset Q(v)$ be such that $u_{n} \rightarrow u \in K$. Hence, one has

$$
\inf _{v^{*} \in G(v)}\left\langle v^{*}+H, v-u_{n}\right\rangle+\phi(v)-\phi\left(u_{n}\right) \geq 0 \text { for all } n \in \mathbb{N} .
$$


Combining this inequaity with the weak lower semicontinuity of $\phi$, we have

$$
\inf _{v^{*} \in G(v)}\left\langle v^{*}+H, v-u\right\rangle+\phi(v)-\phi(u) \geq 0 \text { for all } n \in \mathbb{N} .
$$

So, $Q(v)$ is weakly closed for each $v \in K$. In addition, from the convexity of $\phi$, we can conclude that $Q(v)$ is convex for each $v \in K$.

We further proceed by examining two cases:

(a) the mapping $Q$ is a KKM mapping.

(b) the mapping $Q$ is not a KKM mapping.

Assume that the case (a) occurs, i.e., $Q$ is a KKM mapping. Since $K$ is a bounded, closed and convex subset of a reflexive Banach space $E$, it follows that $K$ is weakly compact, so does $G(v)$ for each $v \in K$. By applying F-KKM theorem, Lemma 2.12, we obtain

$$
\bigcap_{v \in K} G(v) \neq \emptyset \text {. }
$$

We deduce that there is $u \in K$ such that

$$
\left\langle v^{*}+H, v-u\right\rangle+\phi(v)-\phi(u) \geq 0 \text { for all } v^{*} \in G(v) \text { and } v \in K .
$$

Next, for any $v \in K$ and $t \in(0,1)$, letting $v_{t}:=t v+(1-\lambda) u \in K$, we have

$$
\left\langle v_{t}^{*}+H, v_{t}-u\right\rangle+\phi\left(v_{t}\right)-\phi(u) \geq 0 \text { for all } v_{t}^{*} \in G\left(v_{t}\right) .
$$

Similarly as we did before, it follows that

$$
\left\langle v_{t}^{*}+H, v-u\right\rangle+\phi(v)-\phi(u) \geq 0 \text { for all } v_{t}^{*} \in G\left(v_{t}\right) .
$$

By virtue of the upper semicontinuity of $G$, there exists a subsequence of $\left\{v_{t}^{*}\right\}$ such that its limit $u^{*} \in G(u)$. Hence, we have

$$
\left\langle u^{*}+H, v-u\right\rangle+\phi(v)-\phi(u) \geq 0 \text { for all } v \in K,
$$

which implies that $u \in S O L(K, G(\cdot)+H, \phi)$.

Now, suppose that case (b) holds, i.e., $Q$ is not a KKM mapping. By the definition of KKM mapping, there exist a finite sequence $\left\{v_{1}, v_{2}, \ldots, v_{N}\right\} \subset K$ and $u_{0} \in$ $\operatorname{co}\left\{v_{1}, v_{2}, \ldots, v_{N}\right\}$ with $u_{0}:=\sum_{i=1}^{N} \lambda_{i} v_{i}, \lambda_{i} \in[0,1]$ and $\sum_{i=1}^{N} \lambda_{i}=1$ such that

$$
u_{0} \notin \bigcup_{i=1}^{N} Q\left(v_{i}\right) \text {. }
$$

Hence, for each $i \in\{1,2, \ldots, N\}$, it holds

$$
\inf _{v_{i}^{*} \in G\left(v_{i}\right)}\left\langle v_{i}^{*}+H, v_{i}-u_{0}\right\rangle+\phi\left(v_{i}\right)-\phi\left(u_{0}\right)<0 .
$$

Therefore, we assert that

Claim 1 There exists a neighbourhood $U$ of $u_{0}$ such that for all $v \in U \cap K$, we have

$$
\inf _{v_{i}^{*} \in G\left(v_{i}\right)}\left\langle v_{i}^{*}+H, v_{i}-v\right\rangle+\phi\left(v_{i}\right)-\phi(v)<0 \text { for all } i \in\{1,2, \ldots, N\} .
$$

Arguing by contradiction, for any neighbourhood $U$ of $u_{0}$, there are $v_{0} \in U \cap K$ and $i_{0} \in\{1,2, \ldots, N\}$ such that

$$
\inf _{v_{i_{0}}^{*} \in G\left(v_{i_{0}}\right)}\left\langle v_{i_{0}}^{*}+H, v_{i_{0}}-v_{0}\right\rangle+\phi\left(v_{i_{0}}\right)-\phi\left(v_{0}\right) \geq 0 .
$$


In particular, we can choose $U=B\left(u_{0}, \frac{1}{n}\right):=\left\{u \in E \mid\left\|u_{0}-u\right\|_{E} \leq \frac{1}{n}\right\}$. Then, for each $n \in \mathbb{N}$, we are able to take $v_{n} \in U \cap K$ and $i_{n} \in\{1,2, \ldots, N\}$ such that

$$
\inf _{v_{i_{n}}^{*} \in G\left(v_{i_{n}}\right)}\left\langle v_{i_{n}}^{*}+H, v_{i_{n}}-v_{n}\right\rangle+\phi\left(v_{i_{n}}\right)-\phi\left(v_{n}\right) \geq 0 .
$$

Recalling that $i_{n} \in\{1,2, \ldots, N\}$ for all $n \in \mathbb{N}$, without any loss of generality, we may assume that there exists $i_{0}$ such that for all $n \in \mathbb{N}$, it holds

$$
\inf _{v_{i_{0}}^{*} \in G\left(v_{i_{0}}\right)}\left\langle v_{i_{0}}^{*}+H, v_{i_{0}}-v_{n}\right\rangle+\phi\left(v_{i_{0}}\right)-\phi\left(v_{n}\right) \geq 0,
$$

that is

$$
\left\langle v_{i_{0}}^{*}+H, v_{i_{0}}-v_{n}\right\rangle+\phi\left(v_{i_{0}}\right)-\phi\left(v_{n}\right) \geq 0 \text { for all } v_{i_{0}}^{*} \in G\left(v_{i_{0}}\right) .
$$

Passing to the limit as $n \rightarrow \infty$ in the above inequality, it reveals that $v_{n} \rightarrow u_{0}$ and

$$
\left\langle v_{i_{0}}^{*}+H, v_{i_{0}}-u_{0}\right\rangle+\phi\left(v_{i_{0}}\right)-\phi\left(u_{0}\right) \geq 0 \text { for all } v_{i_{0}}^{*} \in G\left(v_{i_{0}}\right) .
$$

Hence

$$
\inf _{v_{i_{0}}^{*} \in G\left(v_{i_{0}}\right)}\left\langle v_{i_{0}}^{*}+H, v_{i_{0}}-u_{0}\right\rangle+\phi\left(v_{i_{0}}\right)-\phi\left(u_{0}\right) \geq 0,
$$

which contradicts condition (3.5), so, Claim 1 is proved.

By applying Claim 1 and using the $\phi$-pseudomonotonicity of $G+H$, we conclude

$$
\left\langle v^{*}+H, v-v_{i}\right\rangle+\phi(v)-\phi\left(v_{i}\right) \geq 0 \text { for all } v^{*} \in G(v) \text { and } v \in U \cap K .
$$

This implies

$$
\left\langle v^{*}+H, v-u_{0}\right\rangle+\phi(v)-\phi\left(u_{0}\right) \geq \sum_{i=1}^{N} \lambda_{i}\left[\left\langle v^{*}+H, v-v_{i}\right\rangle+\phi(v)-\phi\left(v_{i}\right)\right] \geq 0
$$

for all $v^{*} \in G(v)$ and $v \in U \cap K$. Moreover, for any $w \in K$, if $t$ is small enough, we can take $v_{t}:=t w+(1-t) u_{0} \in U \cap K$ in (3.6) to get

$$
\left\langle v_{t}^{*}+H, w-u_{0}\right\rangle+\phi(w)-\phi\left(u_{0}\right) \geq 0 \text { for all } v_{t}^{*} \in G\left(v_{t}\right) .
$$

Since $G$ is upper semicontinuous with compact values, we can assume $v_{t}^{*} \rightarrow u_{0}^{*} \in G\left(u_{0}\right)$. Therefore, we get

$$
\left\langle u_{0}^{*}+H, w-u_{0}\right\rangle+\phi(w)-\phi\left(u_{0}\right) \geq 0 \text { for all } w \in K .
$$

This means $u_{0} \in \operatorname{SOL}(K, G(\cdot)+H, \phi)$.

Moreover, when $K$ is unbounded, we conclude with the following.

Claim 2 There exist $k \in \mathbb{N}$ and $u \in S O L(B(\widetilde{v}, k), G(\cdot)+H, \phi)$ such that $\|u-\widetilde{v}\|_{E}<k$, where $\tilde{v}$ is defined in condition $\left(A_{2}\right)$ and $B(\widetilde{v}, k):=\left\{u \in K \mid\|u-\widetilde{v}\|_{E} \leq k\right\}$.

If the claim does not hold, then for each $k>0$ and for all $u \in S O L(B(\widetilde{v}, k), G(\cdot)+$ $H, \phi)$ one has $\|u-\widetilde{v}\|_{E}=k$. From hypothesis $\left(A_{2}\right)$, if $\|u\|_{E}$ large enough, there exists an increasing function $r: \mathbb{R}_{+} \rightarrow(0,+\infty)$ with $r(k) \rightarrow \infty$, as $k \rightarrow \infty$ such that

$$
\inf _{u^{*} \in G(u)}\left\langle u^{*}, u-\widetilde{v}\right\rangle+\phi(u)-\phi(\widetilde{v}) \geq\|u\|_{E} r\left(\|u\|_{E}\right) .
$$

Hence, for $k$ large enough such that $r\left(k-\|\widetilde{v}\|_{E}\right)>\frac{\|H\|_{E^{*} k}}{\|\widetilde{v}\|_{E}+k}$, we have

$$
\begin{gathered}
\sup _{u^{*} \in G(u)}\left\langle u^{*}+H, \widetilde{v}-u\right\rangle+\phi(\widetilde{v})-\phi(u) \leq\left(\|H\|_{E^{*}}-r\left(k-\|\widetilde{v}\|_{E}\right)\right) k \\
+\|\widetilde{v}\|_{E} r\left(k-\|\widetilde{v}\|_{E}\right)<0 .
\end{gathered}
$$


This is a contradiction, since $u \in \operatorname{SOL}(B(\widetilde{v}, k), G(\cdot)+H, \phi)$. Therefore, Claim 2 is fulfilled.

Now, we assume $k>0$ and $u \in K$ are such that $u \in S O L(B(\widetilde{v}, k), G(\cdot)+H, \phi)$ and $\|u-\widetilde{v}\|_{E}<k$. We will prove that $u \in S O L(K, G(\cdot)+H, \phi)$. For any $v \in K$ and $t \in(0,1)$, we have $v_{t}=t v+(1-t) u \in B(\widetilde{v}, k)$, where $t$ is small enough. Analogously as above, we also get that there exists $u^{*} \in G(u)$ such that

$$
\left\langle u^{*}, v_{t}-u\right\rangle+\phi\left(v_{t}\right)-\phi(u) \geq 0,
$$

and therefore

$$
\left\langle u^{*}, v-u\right\rangle+\phi(v)-\phi(u) \geq 0 \text { for all } v \in K .
$$

This implies that $u \in \operatorname{SOL}(K, G(\cdot)+H, \phi)$, which completes the proof of the theorem.

We observe that Theorem 3.3 extends the recent results obtained by Liu-Zeng-Motreanu [18], Liu-Migórski-Zeng [15], and Wang et al. [36]. In fact, the main approach employed in the proof of Theorem 3.3 is the F-KKM theorem, which was also used in [15]. However, it does not follow the lines of the proof of [15, Lemma 3.1]. In the present proof, the operator is multi-valued not a single-valued one, and we have introduced more general assumptions for the operators.

From the proof of Theorem 3.3, we can see that the condition $\left(A_{2}\right)$ indeed indicates that the multivalued mapping $H \mapsto S O L(K, G(\cdot)+H, \phi)$ for (MQVI) is bounded.

Theorem 3.4 Assume that $\left(A_{1}\right)$ and $\left(A_{2}\right)$ hold. Then the multivalued mapping $H \mapsto$ $\operatorname{SOL}(K, G(\cdot)+H, \phi)$ is bounded.

Proof Arguing by contradiction, we assume that there exists $N_{0}>0$ such that the set $\operatorname{SOL}\left(K, G(\cdot)+B_{E^{*}}\left(0, N_{0}\right), \phi\right)$ is unbounded, where $B_{E^{*}}\left(0, N_{0}\right):=\left\{H \in E^{*} \mid\|H\|_{E^{*}} \leq\right.$ $\left.N_{0}\right\} \subset E^{*}$, that is

$$
\sup _{H \in B_{E^{*}}\left(0, N_{0}\right)}\left\{\|u\|_{E} \mid u \in \operatorname{SOL}(K, G(\cdot)+H, \phi)\right\}=+\infty .
$$

Therefore, we can find sequences $H_{k} \in B_{E^{*}}\left(0, N_{0}\right)$ and $u_{k} \in S O L\left(K, G(\cdot)+H_{k}, \phi\right)$ such that $\left\|u_{k}\right\|_{E}>k$ for each $k=1,2, \ldots$.

It follows from hypothesis $\left(A_{2}\right)$ that there are an increasing function $r: \mathbb{R}_{+} \rightarrow(0,+\infty)$ with $r(k) \rightarrow+\infty$, as $k \rightarrow+\infty$ and a constant $M>0$ such that for each $\|u\|_{E} \geq M$, we have

$$
\inf _{u^{*} \in G(u)}\left\langle u^{*}, u-\widetilde{v}\right\rangle+\phi(u)-\phi(\widetilde{v}) \geq r\left(\|u\|_{E}\right)\|u\|_{E} .
$$

Hence, for $k$ large enough such that $r(k)>N_{0}+\frac{N_{0}\|\widetilde{v}\|_{E}}{k}$, one has

$$
\begin{aligned}
& \sup _{u_{k}^{*} \in G(u)}\left\langle u_{k}^{*}+H_{k}, \tilde{v}-u_{k}\right\rangle+\phi(\widetilde{v})-\phi\left(u_{k}\right) \\
& \leq\left[\left\|H_{k}\right\|_{E^{*}}-r\left(\left\|u_{k}\right\|_{E}\right)\right]\left\|u_{k}\right\|_{E}+\left\|H_{k}\right\|_{E^{*}}\|\widetilde{v}\|_{E} \\
& \quad \leq\left[N_{0}-r\left(\left\|u_{k}\right\|_{E}\right)\right]\left\|u_{k}\right\|_{E}+N_{0}\|\widetilde{v}\|_{E}<0 .
\end{aligned}
$$

This is a contradiction, which completes the proof of the theorem.

Remark 3.5 It follows from Theorems 3.3 and 3.4 that the solution set $\operatorname{SOL}(K, G(\cdot)+$ $H, \phi)$ of (MQVI) is bounded. 
Theorem 3.6 Let $E_{1}$ be a reflexive and separable Banach space and $g:[0, T] \times E_{1} \rightarrow$ $E^{*}$ be a continuous function. Assume that $G: K \rightarrow P\left(E^{*}\right)$ is upper semicontinuou with compact values, and $G(\cdot)+g(t, x)$ is $\phi$-pseudomonotone for all $(t, x) \in[0, T] \times E_{1}$. If $G$ fulfills condition $\left(A_{2}\right)$, then the following statements hold

(i) the multivalued map $U:[0, T] \times E_{1} \rightarrow C b v(K)$ given by

$$
U(t, x):=\{u \in K \mid u \in \operatorname{SOL}(K, g(t, x)+G(\cdot), \phi)\}
$$

is strongly-weakly upper semicontinuous,

(ii) the multifunction $t \mapsto U(t, x)$ is measurable for every $x \in E_{1}$.

Proof We only verify assertion (i), because part (ii) can be obtained following the lines of the proof of [15, Theorem 3.4(ii)]. It follows from Theorems 3.3 and 3.4 that $U(t, x) \in$ $C b v(K)$ for all $(t, x) \in[0, T] \times E_{1}$, therefore, $U$ is well-defined. Moreover, Proposition 2.7 indicates that $U$ is strongly-weakly upper semicontinuous if and only if the set $U^{-}(C)$ given by

$$
U^{-}(C):=\left\{(t, x) \in[0, T] \times E_{1} \mid \operatorname{SOL}(K, g(t, x)+G(\cdot), \phi)\right\},
$$

is strongly closed for each weakly closed set $C \subset E$. So, we only need to verify the strong closedness of $U^{-}(C)$.

Let $\left\{\left(t_{n}, x_{n}\right)\right\} \subset U^{-}(C)$ be such that $\left(t_{n}, x_{n}\right) \rightarrow(t, x)$ in $[0, T] \times E_{1}$. This means that

$$
U\left(t_{n}, x_{n}\right) \cap C \neq \varnothing \text { for each } n \in \mathbb{N} .
$$

Hence, there exist $\left\{u_{n}\right\} \subset K$ and $\left\{u_{n}^{*}\right\} \subset E^{*}$ such that $u_{n}^{*} \in G\left(u_{n}\right)$ and

$$
\left\langle u_{n}^{*}+g\left(t_{n}, x_{n}\right), v-u_{n}\right\rangle+\phi(v)-\phi\left(u_{n}\right) \geq 0 \text { for all } v \in K .
$$

By the same proof as in Theorem 3.3, we have for each $n \in \mathbb{N}$

$$
\left\langle v^{*}+g\left(t_{n}, x_{n}\right), v-u_{n}\right\rangle+\phi(v)-\phi\left(u_{n}\right) \geq 0 \text { for all } v^{*} \in G(v) \text { and } v \in K .
$$

On the other hand, Theorem 3.4 ensures that $\left\{u_{n}\right\}$ is also bounded, thanks to the boundness of $\left\{g\left(t_{n}, x_{n}\right)\right\}$. Without any loss of generality, we may assume $u_{n} \rightarrow u$ in $K$ due to the reflexivity of $E$. Thereby, we readily conclude that

$$
\left\langle v^{*}+g(t, x), v-u\right\rangle+\phi(v)-\phi(u) \geq 0 \text { for all } v^{*} \in G(v) \text { and } v \in K .
$$

Moreover, for any $v \in K$ and $\lambda \in(0,1)$, putting $v_{\lambda}:=\lambda v+(1-\lambda) u \in K$ into (3.8), we get

$$
\left\langle v_{\lambda}^{*}+g(t, x), v-u\right\rangle+\phi(v)-\phi(u) \geq 0 \text { for all } v_{\lambda}^{*} \in G\left(v_{\lambda}\right) \text { and } v \in K .
$$

Since $G$ is upper semicontinuous with compact values, then there exists a subsequence of $\left\{v_{\lambda}{ }^{*}\right\}$, denoted again by the same symbol such that $v_{\lambda}^{*} \rightarrow u^{*} \in G(u)$, as $\lambda \rightarrow 0^{+}$. Hence, we easily get $u^{*} \in G(u)$ and

$$
\left\langle u^{*}+g(t, x), v-u\right\rangle+\phi(v)-\phi(u) \geq 0 \text { for all } v \in K .
$$

This implies that $u \in U(t, x) \cap C$ due to the weak closedness of $C$, and hence $(t, x) \in$ $U^{-}(C)$. The proof is complete. 


\section{Existence results for (FPDVI)}

This section is devoted to explore existence of mild solutions for problem (FPDVI) by using results provided in Section 3. For convenience's sake, we denote by $\mathbb{S}$ the set of mild solutions to (FPDVI), see Definition 2.4, namely,

$$
\mathbb{S}:=\left\{(x, u) \mid x \in C\left(0, T ; E_{1}\right), u:[0, T] \rightarrow K \text { measurable, (2.1) holds for a.e. } t\right\} .
$$

From Theorem 3.6, we know that $t \mapsto U(t, x)$ is measurable for each $x \in E_{1}$ and $x \mapsto$ $U(t, x)$ is strongly-weakly u.s.c. for a.e. $t \in(0, T)$. So, invoking the separability of $E_{1}$, see [24, Theorem 3.17] and [11, Theorem 1.3.1], we conclude that $t \mapsto U(t, x(t))$ admits a measurable selection $l:(0, T) \rightarrow E$ such that

$$
l(t) \in U(t, x(t)) \text { for a.e. } t \in(0, T) .
$$

We assume that $g$ is a continuous function and satisfies the following condition

$\left(A_{3}\right) \quad$ there exists a constant $M_{g}>0$ such that

$$
\sup _{(t, x) \in[0, T] \times E_{1}}\|g(t, x)\|_{E^{*}} \leq M_{g} .
$$

From Theorem 3.4 and condition $\left(A_{3}\right)$, we have

$$
l \in L^{\infty}(0, T ; E) \subset L^{2}(0, T ; E) \text { for each } x \in C\left(0, T ; E_{1}\right) .
$$

Thus, the multivalued mapping $P_{U}: C\left(0, T ; E_{1}\right) \rightarrow L^{2}(0, T ; E)$ defined by

$$
P_{U}(x):=\left\{l \in L^{2}(0, T ; E) \mid l(t) \in U(t, x(t)) \text { for a.e. } t \in(0, T)\right\},
$$

is well-defined for each $x \in C\left(0, T ; E_{1}\right)$.

The following lemma shows that $P_{U}$ is strongly-weakly u.s.c. from $C\left(0, T ; E_{1}\right)$ into $L^{2}(0, T ; E)$. Its proof is similar with the one in [15, Lemma 4.1], however, for completness, we include an independent proof.

Lemma 4.1 Assume hypotheses of Theorem 3.6 and condition $\left(A_{3}\right)$. Then, $P_{U}$ is stronglyweakly u.s.c. from $C\left(0, T ; E_{1}\right)$ into $L^{2}(0, T ; E)$.

Proof We now invoke Proposition 2.10 to verify this lemma.

Indeed, Theorem 3.6 implies that $U$ has weakly compact convex values, so does $P_{U}(x)$. Let $\left\{x_{n}\right\} \subset C\left(0, T ; E_{1}\right)$ be such that $x_{n} \rightarrow x^{*}$ in $C\left(0, T ; E_{1}\right)$ and $l_{n} \in P_{U}\left(x_{n}\right)$. Next, we will show that there exists a subsequence of $\left\{l_{n}\right\}$ which converges to $l^{*} \in P_{U}\left(x^{*}\right)$. Because $U\left([0, T] \times E_{1}\right)$ is uniformly bounded by condition $\left(A_{3}\right)$, we know that the sequence $\left\{l_{n}\right\}$ is also bounded in $L^{2}\left(0, T ; E_{1}\right)$. Hence, we may assume that $l_{n} \rightarrow l^{*}$ in $L^{2}\left(0, T ; E_{1}\right)$. It follows from the Mazur lemma (see e.g. [6]) that there is a sequence $\left\{\bar{l}_{n}\right\}$ of a finite combination of the functions $\left\{l_{i} \mid i \geq n\right\}$ such that

$$
\bar{l}_{n} \rightarrow l^{*} \text { strongly in } L^{2}\left(0, T ; E_{1}\right) .
$$

Further, we may suppose that $\bar{l}_{n}(t) \rightarrow l^{*}(t)$ for a.e. $t \in(0, T)$, as $n \rightarrow \infty$, see e.g. [24, Theorem 2.39].

Recall that $U$ is strongly-weakly u.s.c. and $x_{n} \rightarrow x^{*}$ in $C\left(0, T ; E_{1}\right)$. Hence, for every weak neighborhood $\mathcal{V}_{t}$ of $U\left(t, x^{*}(t)\right)$ there is a strong neighborhood $\mathcal{U}_{t}$ of $x^{*}(t)$ such that

$$
U(t, x) \subset \mathcal{V}_{t} \text { for all } x \in \mathcal{U}_{t} .
$$


This clearly implies that $l^{*} \in P_{U}\left(x^{*}\right)$ (more details, we refer to the proof in [1, Lemma 1.1]). Therefore, by applying Proposition 2.10, we complete the proof of the lemma.

In what follows, we denote by $\mathcal{L}\left(E, E_{1}\right)$ the class of linear and bounded operators from $E$ to $E_{1}$. Let $M_{A}>0$ be the constant such that $\sup _{t \in[0, T]}\left\|e^{A t}\right\| \leq M_{A}$. To prove a result on existence of solutions for (FPDVI), we need two additional assumptions.

$\left(A_{4}\right)$ the operator $B:[0, T] \times E_{1} \rightarrow \mathcal{L}\left(E, E_{1}\right)$ satisfies the Carathéodory conditions, and there exist a constant $\beta \in(0, \alpha)$ and a function $\rho_{B} \in L^{\frac{1}{\beta}}\left(0, T ; \mathbb{R}_{+}\right)$such that

$$
\|B(t, x)\|_{\mathcal{L}\left(E, E_{1}\right)} \leq \rho_{B}(t)\left(1+\|x\|_{E_{1}}\right) \text { for all }(t, x) \in[0, T] \times E_{1},
$$

$\left(A_{5}\right)$ the function $f(\cdot, x):[0, T] \rightarrow E_{1}$ is measurable for all $x \in E_{1}$, and there exists a function $\rho_{f} \in L^{\frac{1}{\beta}}\left(0, T ; \mathbb{R}_{+}\right)$such that $f(t, \cdot): E_{1} \rightarrow E_{1}$ fulfills

$$
\left\{\begin{array}{l}
\|f(t, x)-f(t, y)\|_{E_{1}} \leq \rho_{f}(t)\|x-y\|_{E_{1}} \text { for all } x, y \in E_{1}, \\
\|f(t, 0)\|_{E_{1}} \leq \rho_{f}(t) .
\end{array}\right.
$$

The main result in this paper reads as follows.

Theorem 4.2 Assume hypotheses of Theorem 3.6 and conditions $\left(A_{3}\right)-\left(A_{5}\right)$. In addition, let the operator $e^{A t}, t>0$, be compact. Then, the solution set $\mathbb{S}$ of problem (FPDVI) given by (4.1) is nonempty.

Proof Consider the multivalued mapping $\Omega: C\left(0, T ; E_{1}\right) \rightarrow P\left(C\left(0, T ; E_{1}\right)\right)$ associated with problem (FPDVI) and defined by

$$
\begin{aligned}
\Omega(x):= & \left\{y \in C\left(0, T ; E_{1}\right) \mid y(t)=P_{\alpha}(t) x_{0}+\int_{0}^{t}(t-s)^{\alpha-1} Q_{\alpha}(t-s)\right. \\
& {\left.[B(s, x(s)) l(s)+f(s, x(s))] d s \text { for a.e. } t \in(0, T) \text { and } l \in P_{U}(x)\right\} . }
\end{aligned}
$$

It is obvious that we only need to prove that $\Omega$ has at least one fixed point in $C\left(0, T ; E_{1}\right)$.

First, we prove the following claims.

Claim $3 \Omega$ is a bounded mapping with convex values, and maps bounded sets into equicontinuous sets of $C\left(0, T ; E_{1}\right)$.

It is evident that $\Omega$ has convex values for all $x \in C\left(0, T ; E_{1}\right)$ due to the convexity of $P_{U}(x)$. Next, we will show that $\Omega$ is a bounded mapping. In what follows, we consider the ball

$$
B_{\mathcal{C}}(0, k):=\left\{x \in C\left(0, T ; E_{1}\right) \mid\|x\|_{C\left(0, T ; E_{1}\right)} \leq k\right\}, k>0 .
$$

For any $x \in B_{\mathcal{C}}(0, k)$ and $y \in \Omega(x)$, there exists $l \in P_{U}(x)$ such that

$$
y(t)=P_{\alpha}(t) x_{0}+\int_{0}^{t}(t-s)^{\alpha-1} Q_{\alpha}(t-s)[B(s, x(s)) l(s)+f(s, x(s))] d s
$$

for a.e. $t \in(0, T)$. By the boundedness of $g$ and Theorem 3.4, there exists a constant $M_{g}>0$ such that

$$
\|l(t)\|_{E} \leq M_{g} \text { for a.e. } t \in[0, T] .
$$


From Lemma 2.6, we obtain

$$
\begin{aligned}
\| & y(t) \|_{E_{1}} \\
\leq & \left\|P_{\alpha}(t) x_{0}\right\|_{E_{1}}+\int_{0}^{t}(t-s)^{\alpha-1}\left\|Q_{\alpha}(t-s)[B(s, x(s)) l(s)+f(s, x(s))]\right\|_{E_{1}} d s \\
\leq & M_{A}\left\|x_{0}\right\|_{E_{1}}+\frac{M_{A}}{\Gamma(\alpha)} \int_{0}^{t}(t-s)^{\alpha-1}\left[\|B(s, x(s)) l(s)\|_{E_{1}}+\|f(s, x(s))\|_{E_{1}}\right] d s \\
\leq & M_{A}\left\|x_{0}\right\|_{E_{1}}+\frac{M_{A}}{\Gamma(\alpha)} \int_{0}^{t}(t-s)^{\alpha-1}\left[M_{g} \rho_{B}(s)\left(1+\|x(s)\|_{E_{1}}\right)+\rho_{f}(s)\left(1+\|x(s)\|_{E_{1}}\right)\right] d s \\
\leq & M_{A}\left\|x_{0}\right\|_{E_{1}}+\frac{M_{A}}{\Gamma(\alpha)} \int_{0}^{t}(t-s)^{\alpha-1}\left[M_{g} \rho_{B}(s)\left(1+\|x\|_{\mathcal{C}}\right)+\rho_{f}(s)\left(1+\|x\|_{\mathcal{C}}\right)\right] d s \\
\leq & M_{A}\left\|x_{0}\right\|_{E_{1}}+\frac{M_{A} M_{g}\left(1+\|x\|_{\mathcal{C}}\right)}{\Gamma(\alpha)}\left[\left(\frac{1-\beta}{\alpha-\beta} t^{\gamma}\right)\right]^{1-\beta}\left\|\rho_{B}\right\|_{L^{\frac{1}{\beta}}}+ \\
& +\frac{M_{A}\left(1+\|x\|_{\mathcal{C}}\right)}{\Gamma(\alpha)}\left[\left(\frac{1-\beta}{\alpha-\beta} t^{\gamma}\right)\right]^{1-\beta}\left\|\rho_{f}\right\|_{L^{\frac{1}{\beta}}} \\
\leq & M_{A}\left\|x_{0}\right\|_{E_{1}}+\frac{M_{A} M_{g}(1+k)}{\Gamma(\alpha)}\left[\left(\frac{1-\beta}{\alpha-\beta} T^{\gamma}\right)\right]^{1-\beta}\left\|\rho_{B}\right\|_{L^{\frac{1}{\beta}}}+ \\
& +\frac{M_{A}(1+k)}{\Gamma(\alpha)}\left[\left(\frac{1-\beta}{\alpha-\beta} T^{\gamma}\right)\right]^{1-\beta}\left\|\rho_{f}\right\|_{L^{\frac{1}{\beta}}},
\end{aligned}
$$

where $\gamma:=\frac{\alpha-\beta}{1-\beta}$ and $\|x\|_{\mathcal{C}}:=\sup _{t \in[0, T]}\|x(t)\|_{E_{1}}$. This means that the set $\Omega\left(B_{\mathcal{C}}(0, k)\right)$ is bounded in $C\left(0, T ; E_{1}\right)$, i.e., $\Omega$ is a bounded operator.

It remains to verify that $\Omega\left(B_{\mathcal{C}}(0, k)\right)$ is a set of equicontinuous functions in $C\left(0, T ; E_{1}\right)$. Let $0<s<t<t+h \leq T$ with $h>0$ and $0<\epsilon<t$. For any $x \in B_{\mathcal{C}}(0, k)$ and $y \in \Omega(x)$, there exists $l \in P_{U}$ such that

$$
y(t)=P_{\alpha}(t) x_{0}+\int_{0}^{t}(t-s)^{\alpha-1} Q_{\alpha}(t-s)[B(s, x(s)) l(s)+f(s, x(s))] d s
$$

for a.e. $t \in(0, T)$. Therefore, we have

$$
\begin{aligned}
y(t & +h)-y(t) \\
& =\int_{t}^{t+h}(t+h-s)^{\alpha-1} Q_{\alpha}(t+h-s)[B(s, x(s)) l(s)+f(s, x(s))] d s \\
& +\int_{t-\epsilon}^{t}(t+h-s)^{\alpha-1}\left[Q_{\alpha}(t+h-s)-Q_{\alpha}(t-s)\right][B(s, x(s)) l(s)+f(s, x(s))] d s \\
& +\int_{t-\epsilon}^{t}\left[(t+h-s)^{\alpha-1}-(t-s)^{\alpha-1}\right] Q_{\alpha}(t-s)[B(s, x(s)) l(s)+f(s, x(s))] d s \\
& +\int_{0}^{t-\epsilon}(t+h-s)^{\alpha-1}\left[Q_{\alpha}(t+h-s)-Q_{\alpha}(t-s)\right][B(s, x(s)) l(s)+f(s, x(s))] d s \\
& +\int_{0}^{t-\epsilon}\left[(t+h-s)^{\alpha-1}-(t-s)^{\alpha-1}\right] Q_{\alpha}(t-s)[B(s, x(s)) l(s)+f(s, x(s))] d s \\
& +P_{\alpha}(t+h) x_{0}-P_{\alpha}(t) x_{0} .
\end{aligned}
$$


From the above inequality, we deduce

$$
\|y(t+h)-y(t)\|_{E_{1}} \leq \sum_{i=1}^{6}\left\|I_{i}\right\|_{E_{1}},
$$

where $I_{i}$, for $i=1,2, \ldots, 6$ are defined by

$$
\begin{aligned}
& I_{1}:=P_{\alpha}(t+h) x_{0}-P_{\alpha}(t) x_{0}, \\
& I_{2}:=\int_{t}^{t+h}(t+h-s)^{\alpha-1} Q_{\alpha}(t+h-s)[B(s, x(s)) l(s)+f(s, x(s))] d s, \\
& I_{3}:=\int_{t-\epsilon}^{t}(t+h-s)^{\alpha-1}\left[Q_{\alpha}(t+h-s)-Q_{\alpha}(t-s)\right][B(s, x(s)) l(s)+f(s, x(s))] d s, \\
& I_{4}:=\int_{t-\epsilon}^{t}\left[(t+h-s)^{\alpha-1}-(t-s)^{\alpha-1}\right] Q_{\alpha}(t-s)[B(s, x(s)) l(s)+f(s, x(s))] d s, \\
& I_{5}:=\int_{0}^{t-\epsilon}(t+h-s)^{\alpha-1}\left[Q_{\alpha}(t+h-s)-Q_{\alpha}(t-s)\right][B(s, x(s)) l(s)+f(s, x(s))] d s, \\
& I_{6}:=\int_{0}^{t-\epsilon}\left[(t+h-s)^{\alpha-1}-(t-s)^{\alpha-1}\right] Q_{\alpha}(t-s)[B(s, x(s)) l(s)+f(s, x(s))] d s .
\end{aligned}
$$

We readily get from the compactness of $e^{A t}$ and Lemma 2.6 that

$$
\left\|I_{1}\right\|_{E_{1}} \rightarrow 0, \text { as } h \rightarrow 0 .
$$

For integral $I_{2}$, from hypotheses $\left(A_{4}\right)$ and $\left(A_{5}\right)$, we have

$$
\begin{aligned}
\left\|I_{2}\right\|_{E_{1}} & \\
\leq & \int_{t}^{t+h}(t+h-s)^{\alpha-1}\left\|Q_{\alpha}(t+h-s)[B(s, x(s)) l(s)+f(s, x(s))]\right\|_{E_{1}} d s \\
\leq & \int_{t}^{t+h}(t+h-s)^{\alpha-1}\left\|Q_{\alpha}(t+h-s)\right\|\left[\|B(s, x(s)) l(s)\|_{E_{1}}+\|f(s, x(s))\|_{E_{1}}\right] d s \\
\leq & \frac{M_{A}}{\Gamma(\alpha)} \int_{t}^{t+h}(t+h-s)^{\alpha-1}\left[M_{g} \rho_{B}(s)\left(1+\|x(s)\|_{E_{1}}\right)+\rho_{f}(s)\left(1+\|x(s)\|_{E_{1}}\right)\right] d s \\
\leq & \frac{M_{A}}{\Gamma(\alpha)} \int_{t}^{t+h}(t+h-s)^{\alpha-1}\left[M_{g} \rho_{B}(s)\left(1+\|x\|_{\mathcal{C}}\right)+\rho_{f}(s)\left(1+\|x\|_{\mathcal{C}}\right)\right] d s \\
\leq & \frac{M_{A}}{\Gamma(\alpha)}\left\{M_{g}(1+k)\left[\left(\frac{1-\beta}{\alpha-\beta} h^{\gamma}\right)\right]^{1-\beta}\left\|\rho_{B}\right\|_{L^{\frac{1}{\beta}}}+\right. \\
& \left.+(1+k)\left[\left(\frac{1-\beta}{\alpha-\beta} h^{\gamma}\right)\right]^{1-\beta}\left\|\rho_{f}\right\|_{L^{\frac{1}{\beta}}}\right\} \rightarrow 0, \text { as } h \rightarrow 0 .
\end{aligned}
$$


As concerns integral $I_{3}$, we calculate

$\left\|I_{3}\right\|_{E_{1}}$

$$
\begin{aligned}
\leq & \int_{t-\epsilon}^{t}(t+h-s)^{\alpha-1}\left\|Q_{\alpha}(t+h-s)-Q_{\alpha}(t-s)\right\|\|B(s, x(s)) l(s)+f(s, x(s))\|_{E_{1}} d s \\
\leq & \frac{2 M_{A}}{\Gamma(\alpha)} \int_{t-\epsilon}^{t}(t+h-s)^{\alpha-1}\left[M_{g} \rho_{B}(s)\left(1+\|x(s)\|_{E_{1}}\right)+\rho_{f}(s)\left(1+\|x(s)\|_{E_{1}}\right)\right] d s \\
\leq & \frac{2 M_{A}}{\Gamma(\alpha)} \int_{t-\epsilon}^{t}(t+h-s)^{\alpha-1}\left[M_{g} \rho_{B}(s)\left(1+\|x\|_{\mathcal{C}}\right)+\rho_{f}(s)\left(1+\|x\|_{\mathcal{C}}\right)\right] d s \\
\leq & \frac{2 M_{A}}{\Gamma(\alpha)}\left\{M_{g}(1+k)\left[\left(\frac{1-\beta}{\alpha-\beta}\left[(h+\epsilon)^{\gamma}-h^{\gamma}\right]\right)\right]^{1-\beta}\left\|\rho_{B}\right\|_{L^{\frac{1}{\beta}}}+\right. \\
& \left.+(1+k)\left[\left(\frac{1-\beta}{\alpha-\beta}\left[(h+\epsilon)^{\gamma}-h^{\gamma}\right]\right)\right]^{1-\beta}\left\|\rho_{f}\right\|_{L^{\frac{1}{\beta}}}\right\} \\
\leq & \frac{2 M_{A}}{\Gamma(\alpha)}\left\{M_{g}(1+k)\left[\left(\frac{1-\beta}{\alpha-\beta} 2 h^{\gamma}\right)\right]^{1-\beta}\left\|\rho_{B}\right\|_{L^{\frac{1}{\beta}}}\right. \\
& \left.+(1+k)\left[\left(\frac{1-\beta}{\alpha-\beta} 2 h^{\gamma}\right)\right]^{1-\beta}\left\|\rho_{f}\right\|_{L^{\frac{1}{\beta}}}\right\} \rightarrow 0, \text { as } h \rightarrow 0 .
\end{aligned}
$$

For integral $I_{4}$, we can calculate

$$
\begin{aligned}
& \left\|I_{4}\right\|_{E_{1}} \\
& \leq \int_{t-\epsilon}^{t}\left|(t+h-s)^{\alpha-1}-(t-s)^{\alpha-1}\right|\left\|Q_{\alpha}(t-s)[B(s, x(s)) l(s)+f(s, x(s))]\right\|_{E_{1}} d s \\
& \leq \frac{M_{A}}{\Gamma(\alpha)} \int_{t-\epsilon}^{t}\left|(t+h-s)^{\alpha-1}-(t-s)^{\alpha-1}\right|\left(M_{g} \rho_{B}(s)+\rho_{f}(s)\right)\left(1+\|x(s)\|_{E_{1}}\right) d s \\
& \leq \frac{M_{A}}{\Gamma(\alpha)} \int_{t-\epsilon}^{t}\left|(t+h-s)^{\alpha-1}-(t-s)^{\alpha-1}\right|\left(M_{g} \rho_{B}(s)+\rho_{f}(s)\right)\left(1+\|x\|_{\mathcal{C}}\right) d s \\
& \leq \frac{M_{A}}{\Gamma(\alpha)}\left(M_{g}\left(1+\|x\|_{\mathcal{C}}\right)\left(\int_{t-\epsilon}^{t}\left|(t+h-s)^{\alpha-1}-(t-s)^{\alpha-1}\right|^{\frac{1}{1-\beta}} d s\right)^{1-\beta}\left\|\rho_{B}\right\|_{L^{\frac{1}{\beta}}}\right. \\
& \left.+\left(1+\|x\|_{\mathcal{C}}\right)\left(\int_{t-\epsilon}^{t}\left|(t+h-s)^{\alpha-1}-(t-s)^{\alpha-1}\right|^{\frac{1}{1-\beta}} d s\right)^{1-\beta}\left\|\rho_{f}\right\|_{L^{\frac{1}{\beta}}}\right) \\
& \leq \frac{M_{A}}{\Gamma(\alpha)}\left(M_{g}\left(1+\|x\|_{\mathcal{C}}\right)\right)\left(\int_{t-\epsilon}^{t}\left|(t+h-s)^{\gamma-1}-(t-s)^{\gamma-1}\right| d s\right)^{1-\beta}\left\|\rho_{B}\right\|_{L^{\frac{1}{\beta}}} \\
& \left.+\left(1+\|x\|_{\mathcal{C}}\right)\left(\int_{t-\epsilon}^{t}\left|(t+h-s)^{\gamma-1}-(t-s)^{\gamma-1}\right| d s\right)^{1-\beta}\left\|\rho_{f}\right\|_{L^{\frac{1}{\beta}}}\right) \\
& \leq \frac{M_{A}}{\Gamma(\alpha)}\left(\frac{M_{g}\left(1+\|x\|_{\mathcal{C}}\right)}{\gamma^{1-\beta}}\left|(h+\epsilon)^{\gamma}-h^{\gamma}-\epsilon^{\gamma}\right|^{1-\beta}\left\|\rho_{B}\right\|_{L^{\frac{1}{\beta}}}\right.
\end{aligned}
$$




$$
\begin{aligned}
& \left.+\frac{\left(1+\|x\|_{\mathcal{C}}\right)}{\gamma^{1-\beta}}\left|(h+\epsilon)^{\gamma}-h^{\gamma}-\epsilon^{\gamma}\right|^{1-\beta}\left\|\rho_{f}\right\|_{L^{\frac{1}{\beta}}}\right) \\
\leq & \frac{M_{A}}{\Gamma(\alpha)}\left(\frac{M_{g}(1+k)}{\gamma^{1-\beta}}(3 h)^{\gamma(1-\beta)}\left\|\rho_{B}\right\|_{L^{\frac{1}{\beta}}}+\frac{(1+k)}{\gamma^{1-\beta}}(3 h)^{\gamma(1-\beta)}\left\|\rho_{f}\right\|_{L^{\frac{1}{\beta}}}\right) \rightarrow 0,
\end{aligned}
$$

as $h \rightarrow 0$. An application of Lemma 2.6 yields

$\left\|I_{5}\right\|_{E_{1}}$

$$
\begin{aligned}
\leq & \int_{0}^{t-\epsilon}(t+h-s)^{\alpha-1}\left\|Q_{\alpha}(t+h-s)-Q_{\alpha}(t-s)\right\|\|B(s, x(s)) l(s)+f(s, x(s))\|_{E_{1}} d s \\
\leq & \sup _{s \in[0, t-\epsilon]}\left\|Q_{\alpha}(t+h-s)-Q_{\alpha}(t-s)\right\| \int_{0}^{t-\epsilon}(t+h-s)^{\alpha-1}\left(M_{g} \rho_{B}(s)\left(1+\|x(s)\|_{E_{1}}\right)\right. \\
& \left.\quad+\rho_{f}(s)\left(1+\|x(s)\|_{E_{1}}\right)\right) d s \\
\leq & \sup _{s \in[0, t-\epsilon]}\left\|Q_{\alpha}(t+h-s)-Q_{\alpha}(t-s)\right\|\left(M _ { g } \| \rho _ { B } \| _ { L ^ { \frac { 1 } { \beta } } } ( 1 + k ) \left(\frac{1-\beta}{\alpha-\beta} \mid(t+h)^{\gamma}\right.\right. \\
& \left.\left.-(\epsilon+h)^{\gamma} \mid\right)^{1-\beta}+\left\|\rho_{f}\right\|_{L^{\frac{1}{\beta}}}(1+k)\left(\frac{1-\beta}{\alpha-\beta}\left|(t+h)^{\gamma}-(\epsilon+h)^{\gamma}\right|\right)^{1-\beta}\right) \\
\rightarrow & 0, \text { as } h \rightarrow 0 .
\end{aligned}
$$

Moreover, for integral $I_{6}$, we estimate

$\left\|I_{6}\right\|_{E_{1}}$

$$
\begin{aligned}
\leq & \int_{0}^{t-\epsilon}\left|(t+h-s)^{\alpha-1}-(t-s)^{\alpha-1}\right|\left\|Q_{\alpha}(t-s)[B(s, x(s)) l(s)+f(s, x(s))]\right\|_{E_{1}} d s \\
\leq & \frac{M_{A}}{\Gamma(\alpha)} \int_{0}^{t-\epsilon}\left|(t+h-s)^{\alpha-1}-(t-s)^{\alpha-1}\right|\left(M_{g} \rho_{B}(s)+\rho_{f}(s)\right)\left(1+\|x(s)\|_{E_{1}}\right) d s \\
\leq & \frac{M_{A}}{\Gamma(\alpha)} \int_{0}^{t-\epsilon}\left|(t+h-s)^{\alpha-1}-(t-s)^{\alpha-1}\right|\left(M_{g} \rho_{B}(s)+\rho_{f}(s)\right)\left(1+\|x\|_{\mathcal{C}}\right) d s \\
\leq & \frac{M_{A}}{\Gamma(\alpha)}\left[M_{g}\left\|\rho_{B}\right\|_{L^{\frac{1}{\beta}}}\left(1+\|x\|_{\mathcal{C}}\right)\left(\int_{0}^{t-\epsilon}\left[(t+h-s)^{\alpha-1}-(t-s)^{\alpha-1}\right]^{\frac{1}{1-\beta}} d s\right)^{1-\beta}\right. \\
& \left.+\left(1+\|x\|_{\mathcal{C}}\right)\left\|\rho_{f}\right\|_{L^{\frac{1}{\beta}}}\left(\int_{0}^{t-\epsilon}\left|(t+h-s)^{\alpha-1}-(t-s)^{\alpha-1}\right|^{\frac{1}{1-\beta}} d s\right)^{1-\beta}\right] \\
\leq & \frac{M_{A}}{\Gamma(\alpha)}\left[M_{g}\left\|\rho_{B}\right\|_{L^{\frac{1}{\beta}}}\left(1+\|x\|_{\mathcal{C}}\right)\left(\int_{0}^{t-\epsilon}\left|(t+h-s)^{\gamma-1}-(t-s)^{\gamma-1}\right| d s\right)^{1-\beta}\right. \\
& \left.+\left(1+\|x\|_{\mathcal{C}}\right)\left\|\rho_{f}\right\|_{L^{\frac{1}{\beta}}}\left(\int_{0}^{t-\epsilon}\left|(t+h-s)^{\gamma-1}-(t-s)^{\gamma-1}\right| d s\right)^{1-\beta}\right] \\
\leq & \frac{M_{A}}{\Gamma(\alpha)}\left[\frac{M_{g}\left\|\rho_{B}\right\|_{L^{\frac{1}{\beta}}}\left(1+\|x\|_{\mathcal{C}}\right)}{\gamma^{1-\beta}}\left(\left|(t+h)^{\gamma}-(h+\epsilon)^{\gamma}-t^{\gamma}+\epsilon^{\gamma}\right|\right)^{1-\beta}\right.
\end{aligned}
$$




$$
\begin{aligned}
& \left.+\frac{\left(1+\|x\|_{\mathcal{C}}\right)\left\|\rho_{f}\right\|_{L^{\frac{1}{\beta}}}}{\gamma^{1-\beta}}\left(\left|(t+h)^{\gamma}-(h+\epsilon)^{\gamma}-t^{\gamma}-\epsilon^{\gamma}\right|\right)^{1-\beta}\right] \\
\leq & \frac{M_{A}}{\Gamma(\alpha)}\left(\frac{M_{g}\left\|\rho_{B}\right\|_{L^{\frac{1}{\beta}}}(1+k)}{\gamma^{1-\beta}}(3 h)^{\gamma(1-\beta)}+\frac{(1+k)\left\|\rho_{f}\right\|_{L^{\frac{1}{\beta}}}}{\gamma^{1-\beta}}(3 h)^{\gamma(1-\beta)}\right) \\
\rightarrow & 0, \text { as } h \rightarrow 0 .
\end{aligned}
$$

Combining estimates (4.3)-(4.8), we conclude

$$
\|y(t+h)-y(t)\|_{E_{1}} \rightarrow 0, \quad \text { as } h \rightarrow 0
$$

for all $y \in \Omega(x)$ and $x \in C\left(0, T ; E_{1}\right)$. This implies that $\Omega\left(B_{\mathcal{C}}(0, k)\right)$ is equicontinuous in $C\left(0, T ; E_{1}\right)$.

From Claim 3 and the Arzela-Ascoli theorem, see [38], it is clear that the mapping $\Omega$ is compact, in particular, it is quasicompact (see Definition 2.8).

Claim $4 \Omega$ is a closed mapping.

Let $\left\{x_{n}\right\},\left\{y_{n}\right\}$ be such that $x_{n} \rightarrow x$ and $y_{n} \rightarrow y$ in $C\left(0, T ; E_{1}\right)$ with $y_{n} \in \Omega\left(x_{n}\right)$. We shall check that $y \in \Omega(x)$. For each $n \in \mathbb{N}$, there exists $l_{n} \in P_{U}\left(x_{n}\right)$ such that

$$
y_{n}(t)=P_{\alpha}(t) x_{0}+\int_{0}^{t}(t-s)^{\alpha-1} Q_{\alpha}\left[B\left(s, x_{n}(s)\right) l_{n}(s)+f\left(s, x_{n}(s)\right)\right] d s
$$

for a.e. $t \in(0, T)$. From Theorem 3.6, we can see that $\left\{l_{n}\right\}$ is bounded in $L^{2}(0, T ; E)$. Therefore, without any loss of generality, we may assume $l_{n} \rightarrow l^{*}$ weakly in $L^{2}(0, T ; E)$. Recall that $P_{U}$ is strongly-weakly upper semicontinuous, see Lemma 4.1, hence $l^{*} \in$ $P_{U}(x)$. On the other hand, we use the compactness of $e^{A t}$, hypotheses $\left(A_{4}\right),\left(A_{5}\right)$ and Lemma 2.6(iii) to obtain

$$
y(t)=P_{\alpha}(t) x_{0}+\int_{0}^{t}(t-s)^{\alpha-1} Q_{\alpha}(t-s)\left[B(s, x(s)) l^{*}(s)+f(s, x(s))\right] d s
$$

for a.e. $t \in(0, T)$ with $l^{*} \in P_{U}(x)$. This implies that $y \in \Omega(x)$, and thus $\Omega$ is a closed operator.

Claim 5 There exists a constant $M_{R}>0$ such that $\Omega$ maps the ball

$$
\widetilde{B}_{\mathcal{C}}\left(0, M_{R}\right):=\left\{x \in C\left(0, T ; E_{1}\right) \mid\|x\|_{*} \leq M_{R}\right\}
$$

into itself, where $\|\cdot\|_{*}$ is the equivalent norm on the space $C\left(0, T ; E_{1}\right)$ given by

$$
\|x\|_{*}:=\max _{t \in[0, T]} e^{-L t}\|x(t)\|_{E_{1}}
$$

with $L>0$ such that

$$
\frac{M_{A}}{\Gamma(\alpha)} \int_{0}^{t} e^{-L(t-s)}(t-s)^{\alpha-1}\left[\rho_{B}(s)+\rho_{f}(s)\right] d s<1 \text { for all } t \in[0, T] .
$$


From (4.9), we can find $M_{R}>0$ such that

$$
\begin{aligned}
& M_{A}\left[\left\|x_{0}\right\|_{E_{1}}+\frac{M_{\|g\|}\left\|\rho_{B}\right\|}{\Gamma(\alpha)} L^{\frac{1}{\beta}}\left(\frac{1-\beta}{\alpha-\beta} T^{\gamma}\right)^{1-\beta}+\frac{\left\|\rho_{f}\right\|_{L^{\frac{1}{\beta}}}}{\Gamma(\alpha)}\left(\frac{1-\beta}{\alpha-\beta} T^{\gamma}\right)^{1-\beta}\right] \\
& \quad+\frac{M_{A} M_{R}}{\Gamma(\alpha)} \int_{0}^{t} e^{-L(t-s)}\left[\rho_{B}(s)+\rho_{f}(s)\right] d s \leq M_{R} .
\end{aligned}
$$

We now prove that $\Omega\left(\widetilde{B}_{\mathcal{C}}\left(0, M_{R}\right)\right) \subseteq \widetilde{B}_{\mathcal{C}}\left(0, M_{R}\right)$. For any $y \in \Omega(x)$ with $x \in \widetilde{B}_{\mathcal{C}}\left(0, M_{R}\right)$, there exists $l \in P_{U}(x)$ such that

$$
y(t)=P_{\alpha}(t) x_{0}+\int_{0}^{t}(t-s)^{\alpha-1} Q_{\alpha}(t-s)[B(s, x(s)) l(s)+f(s, x(s))] d s
$$

for a.e. $t \in(0, T)$. Hence, we calculate

$$
\begin{aligned}
& e^{-L t}\|y(t)\|_{E_{1}} \\
&=e^{-L t}\left\|P_{\alpha}(t) x_{0}+\int_{0}^{t}(t-s)^{\alpha-1} Q_{\alpha}(t-s)[B(s, x(s)) l(s)+f(s, x(s))] d s\right\|_{E_{1}} \\
& \leq M_{A}\left\|x_{0}\right\|_{E_{1}}+\frac{M_{A}\left(1+\|x\|_{*}\right)}{\Gamma(\alpha)} \int_{0}^{t} e^{-L(t-s)}(t-s)^{\alpha-1}\left[M_{g} \rho_{f}(s)+\rho_{f}(s)\right] d s \\
& \leq M_{A}\left[\left\|x_{0}\right\|_{E_{1}}+\frac{M_{g}\left\|\rho_{B}\right\|_{L^{\frac{1}{\beta}}}}{\Gamma(\alpha)}\left(\frac{1-\beta}{\alpha-\beta} T^{\gamma}\right)^{1-\beta}+\frac{\left\|\rho_{f}\right\|_{L^{\frac{1}{\beta}}}}{\Gamma(\alpha)}\left(\frac{1-\beta}{\alpha-\beta} T^{\gamma}\right)^{1-\beta}\right] \\
& \quad+\frac{M_{A}\|x\|_{*}}{\Gamma(\alpha)} \int_{0}^{t} e^{-L(t-s)}(t-s)^{\alpha-1}\left(\rho_{B}(s)+\rho_{f}(s)\right) d s \\
& \leq M_{A}\left[\left\|x_{0}\right\|_{E_{1}}+\frac{M_{g}\left\|\rho_{B}\right\|}{\Gamma(\alpha)}\left(\frac{1-\beta}{\alpha-\beta} T^{\gamma}\right)^{1-\beta}+\frac{\left\|\rho_{f}\right\|_{L^{\frac{1}{\beta}}}}{\Gamma(\alpha)}\left(\frac{1-\beta}{\alpha-\beta} T^{\gamma}\right)^{1-\beta}\right]+ \\
&+\frac{M_{A} M_{R}}{\Gamma(\alpha)} \int_{0}^{t} e^{-L(t-s)}(t-s)^{\alpha-1}\left(\rho_{B}(s)+\rho_{f}(s)\right) d s \\
& \leq M_{R} .
\end{aligned}
$$

This implies $\|y\|_{*} \leq M_{R}$ for all $y \in \Omega\left(\widetilde{B}_{\mathcal{C}}\left(0, M_{R}\right)\right)$, so, $\Omega$ maps $\widetilde{B}_{\mathcal{C}}\left(0, M_{R}\right)$ into itself.

Through Claims 3 and 4 , we can see that the function $\Omega$ has compact and convex values. Moreover, invoking Theorem 2.9 and Claim 4, we obtain that $\Omega$ is upper semicontinuous. On the other hand, by applying Claims 3 and 5 , we are able to find that $\Omega\left(\widetilde{B}_{\mathcal{C}}\left(0, M_{R}\right)\right)$ is relatively compact with $\Omega\left(\widetilde{B}_{\mathcal{C}}\left(0, M_{R}\right)\right) \subset \widetilde{B}_{\mathcal{C}}\left(0, M_{R}\right)$. It turns out that Theorem 2.11 can be applied by taking $G:=\Omega$ in its statement. We deduce that the set of fixed points of mapping $\Omega$ is nonempty. Thus the solution set of (FPDVI) in the sense of Definition 2.4 is nonempty. This completes the proof.

It is worth to mention that in [12] the authors used the theory of measure of noncompactness and a fixed point theorem of condensing multivalued mappings to show the solvability of fractional differential variational inequality in finite dimensional spaces. In this paper, we have applied the theory of semigroups, the Bohnenblust-Karlin fixed point principle for multivalued mappings to verify the existence of solution for a class of generalized fractional differential variational inequalities. The main result of the present paper, Theorem 4.2, extends the recent ones provided in [14, Theorem 4.1] and [36, Lemma 3.3]. Furthermore, 
we also note that the proof of Theorem 4.2 is different than the one given in [15, Theorem 4.2] since it is based on the Bohnenblust-Karlin fixed point principle for multivalued mappings and the theory of fractional calculus.

\section{Conclusion}

In this paper, a new class of generalized fractional differential variational inequalities in Banach spaces is introduced and studied. This class is much more general and complex than the ones considered by Li-Huang-O'Regan [14], Wang-Li-Li-Huang [36], Liu-ZengMotreanu [18], and Liu-Migórski-Zeng [15], etc. We have found suitable conditions under which we have established an existence theorem for the system by using the theory of operator semigroups, the Bohnenblust-Karlin fixed point principle for multivalued mappings, and theory of fractional operators.

Acknowledgements The authors would like to express their thanks to the Editors and the Reviewers for their helpful comments and advices.

Open Access This article is distributed under the terms of the Creative Commons Attribution 4.0 International License (http://creativecommons.org/licenses/by/4.0/), which permits unrestricted use, distribution, and reproduction in any medium, provided you give appropriate credit to the original author(s) and the source, provide a link to the Creative Commons license, and indicate if changes were made.

\section{References}

1. Barbu, V.: Analysis and Control of Nonlinear Infinite Dimensional Systems. Academic Press, Boston (1993)

2. Bohnenblust, H.F., Karlin, S.: On a theorem of ville. In: Contributions to the Theory of Games. Princeton University Press, Princeton (1950)

3. Bothe, D.: Multivalued perturbations of $m$-accretive differential inclusions. Israel J. Math. 108, 109-138 (1998)

4. Chen, X.J., Wang, Z.Y.: Convergence of regularized time-stepping methods for differential variational inequalities. SIAM J. Optim. 23, 1647-1671 (2013)

5. Chen, X.J., Wang, Z.Y.: Differential variational inequality approach to dynamic games with shared constraints. Math. Program. 146, 379-408 (2014)

6. Ekeland, I., Teman, R.: Convex Analysis and Variational Problems. North holland, Amsterdam (1976)

7. Fan, K.: Some properties of convex sets related to fixed point theorems. Math. Ann. 266, 519-537 (1984)

8. Gwinner, J.: On a new class of differential variational inequalities and a stability result. Math. Program. 139, 205-221 (2013)

9. Gwinner, J.: On the $p$-version approximation in the boundary element method for a variational inequality of the second kind modelling unilateral contact and given friction. Appl. Numer. Math. 59, 2774-2784 (2009)

10. Gwinner, J.: Hp-FEM convergence for unilateral contact problems with Tresca friction in plane linear elastostatics. J. Comput. Appl. Math. 254, 175-184 (2013)

11. Kamenskii, M., Obukhovskii, V., Zecca, P.: Condensing Multivalued Maps and Semilinear Differential Inclusions in Banach Space. Water de Gruyter, Berlin (2001)

12. Ke, T.D., Loi, N.V., Obukhovskii, V.: Decay solutions for a class of fractional differential variational inequalities. Fract. Calc. Appl. Anal. 18, 531-553 (2015)

13. Kilbas, A.A., Srivastava, H.M., Trujillo, J.J.: Theory and applications of fractional differential equations. In: North-Holland Mathematics Studies. Elsevier Science B.V., Amsterdam (2006)

14. Li, X.S., Huang, N.J., O'Regan, D.: Differential mixed variational inequalities in finite dimensional spaces. Nonlinear Anal. 72, 3875-3886 (2010) 
15. Liu, Z.H., Migórski, S., Zeng, S.D.: Partial differential variational inequalities involving nonlocal boundary conditions in Banach spaces. J. Differential Equations 263, 3989-4006 (2017)

16. Liu, Z.H., Zeng, S.D., Bai, Y.R.: Maximum principles for multi term space-time variable-order fractional diffusion equations and their applications. Fract. Calc. Appl. Anal. 19, 188-211 (2016)

17. Liu, Z.H., Zeng, S.D.: Differential variational inequalities in infinite Banach spaces. Acta. Math. Sci. 37, 26-32 (2017)

18. Liu, Z.H., Zeng, S.D., Motreanu, D.: Evolutionary problems driven by variational inequalities. J. Differential Equations 260, 6787-6799 (2016)

19. Liu, Z.H., Loi, N.V., Obukhovskii, V.: Existence and global bifurcation of periodic solutions to a class of differential variational inequalities. Int. J. Bifurcation Chaos 23, ID 1350125 (2013)

20. Liu, Z.H., Motreanu, D., Zeng, S.D.: Nonlinear evolutionary systems driven by mixed variational inequalities and its applications. Nonlinear Anal. 42, 409-421 (2018)

21. Liu, Z.H., Zeng, S.D., Motreanu, D.: Partial differential hemivariational inequalities. Adv. Nonlinear Anal. 7, 571-586 (2018)

22. Migórski, S., Zeng, S.D.: A class of differential hemivariational inequalities in Banach spaces. J. Glob Optim (2018)

23. Migórski, S., Zeng, S.D.: Mixed variational inequalities driven by fractional evolutionary equations. Acta Math. Sci. accepted (2018)

24. Migórski, S., Ochal, A., Sofonea, M.: Nonlinear Inclusions and Hemivariational Inequalities, Models and Analysis of Contact Problems Advances in Mechanics and Mathematics 26. Springer, New York (2013)

25. Pang, J.S., Stewart, D.E.: Differential variational inequalities. Math. Program. 113, 345-424 (2008)

26. Podlubny, I.: Fractional Differential Equations. Academic Press, San Diego (1999)

27. Pang, J.S., Stewart, D.E.: Solution dependence on initial conditions in differential variational inequalities. Math. Program. 116, 429-460 (2009)

28. Samko, S.G., Marichev, A.A., Kilbas O.I.: Fractional Integrals and Derivatives: Theory and Applications. Gordon and Breach, New York (1993)

29. Shen, J.L., Pang, J.S.: Linear complementarity systems: Zeni states. SIAM J. Control Optim. 44, 1040 1066 (2005)

30. Su, C.H., Sehgal, V.M.: Some fixed point theorems for condensing multi functions in locally convex spaces. Proc. Natl. Acad. Sci. USA 50, 150-154 (1975)

31. Van, N.T., Ke, T.D.: Asymptotic behavaior of solutions to a class of differential variational inequalities. Ann. Polon. Math. 114, 147-164 (2015)

32. Wang, J.R., Zhou, Y.: Existence and controllability results for fractional semilinear differential inclusions. Nonlinear Anal. 12, 3642-3653 (2011)

33. Wang, X., Huang, N.J.: A class of differential vector variational inequalities in finite dimensional spaces. J. Optim. Theory Appl. 162, 633-648 (2014)

34. Wang, X., Huang, N.J.: Differential vector variational inequalities in finite-dimensional spaces. J. Optim. Theory Appl. 158, 109-129 (2012)

35. Wang, X., Qi, Y.W., Tao, C.Q., Xiao, Y.B.: A class of delay differential variational inequalities. J. Optim. Theory Appl. 172, 56-69 (2017)

36. Wang, X., Li, W., Li, X.S., Huang, N.J.: Stability for differential mixed variational inequalities. Optim. Lett. 8, 1873-1887 (2014)

37. Wang, X., Tang, G.J., Li, X.S., Huang, N.J.: Differential quasi-variational inequalities in finite dimensional spaces. Optimization 64, 895-907 (2015)

38. Zeidler, E.: Nonlinear Functional Analysisc and its Applications, vol. II/B, Nonlinear Monotone Operators. Springer, New York (1990)

39. Zeng, S.D., Baleanu, D., Bai, Y.R., Wu, G.C.: Fractional differential equations of Caputo-Katugampola type and numerical solutions. Appl. Math. Comput. 315, 549-554 (2017)

40. Zeng, S.D., Migórski, S.: A class of time-fractional hemivariational inequalities with application to frictional contact problem. Commun. Nonlinear Sci. 56, 34-48 (2018)

41. Zeng, S.D., Liu, Z.H., Migórski, S.: A class of fractional differential hemivariational inequalities with application to contact problem. Z. Angew. Math. Phys. 69(36), 23 (2018)

42. Zhou, Y., Jiao, F.: Existence of mild solutions for fractional neutral evolution equations. Comput. Math. Appl. 59, 1063-1077 (2010) 